\title{
LA FORMACIÓN DOCENTE DESDE LA NORMATIVIDAD ${ }^{1}$
}

\author{
TEACHER TRAINING FROM REGULATIONS
}

\author{
Sonia López Domínguez ${ }^{2}$ \\ José Alfonso Martín Reyes ${ }^{3}$ \\ Juan Manuel Carreño Cardozo ${ }^{4}$ \\ Edgar Andrés Díaz Velasco ${ }^{5}$
}

\section{Resumen}

El proyecto que da origen a este artículo surge de la unión entre dos grupos de investigación de la Facultad de Educación Física: uno de la Licenciatura en Recreación y Turismo, denominado Lúdica, Cuerpo y Sociedad, y el otro, de la Licenciatura en Educación Física, denominado Estudios en Educación y Experiencia Corporal. Esta investigación tiene por objeto estudiar las Líneas de formación docente de las dos licenciaturas. En este escrito se presenta un estudio de las normas que entre 1998 y 2014 han regulado los diferentes programas de formación docente en las instituciones de educación superior; se escoge dicho periodo teniendo en cuenta que es justo desde 1998 cuando el Ministerio de Educación se propone la tarea de vigilar y supervisar los programas académicos de formación docente, dados el auge y la creación de varios de ellos.

Palabras clave: formación docente, Educación Física, recreación, investigación, normatividad.

\section{Abstract}

The project which gave origin to this article arose from the union between two research groups of the Faculty of Physical Education, one of them, from the degree in Recreation and Tourism, called: Ludic, body and society, and another from the degree in Physical Education: Studies in education and bodily experience. This research aims to study the lines of teacher training in both programs. In this text we present the study about norms that have regulated the various teacher formation programs in institutions of higher education between 1998 and 2014. This period was chosen taking into account that it was in 1998 when the Ministry of Education proposes itself the task of watching and supervising the academic training programs because of the boom and the creation of several of these programs.

Keywords: teacher training, Physical Education, recreation, research, regulations.

Fecha de recepción: 2 de diciembre de 2015

Fecha de aprobación: 3 de mayo de 2016

Para citar este artículo:

López, S., Martín, J. A., Carreño, J. M. \& Díaz, E. A. (2016). La formación docente desde la normatividad. Lúdica Pedagógica, $24,23-32$. Universidad Pedagógica Nacional (CIUP). Investigador principal: Juan Manuel Carreño Cardozo. Grupo de investigación Lúdica, Cuerpo y Sociedad y grupo de investigación en Estudios y Experiencia corporal.

2 Magistra en Educación y Desarrollo Social. Especialista en investigación sobre el fenómeno de las drogas y en docencia universitaria. Licenciada en Educación Física. Docente de planta de la Facultad de Educación Física, Universidad Pedagógica Nacional. Correo electrónico: solopez@ pedagogica.edu.co

3 Magíster en Filosofía. Especialista en investigación social y entrenamiento deportivo. Licenciado en Educación Física. Docente de planta de la Facultad de Educación Física, Universidad Pedagógica Nacional. Correo electrónico: alfonsom@pedagogica.edu.co

4 Magíster en Investigación Social Interdisciplinaria. Especialista en investigación social. Licenciado en Educación Física. Docente de planta. Coordinador del programa Licenciatura en Recreación y Turismo, Facultad de Educación Física, Universidad Pedagógica Nacional. Correo electrónico: jcarreno@pedagogica.edu.co

5 Magíster en Filosofía. Licenciado en Educación Física. Docente ocasional de tiempo completo, Licenciatura en Recreación y Turismo, Facultad de Educación Física, Universidad Pedagógica Nacional. Correo electrónico: andresdiazvelasco@gmail.com 
La metodología que nos proponemos para el desarrollo de esta investigación parte de revisar los documentos de cada una de las dos licenciaturas que hacen parte del trabajo investigativo y de algunas acciones que puedan dar cuenta del estado de la formación docente en ellas, así como hacer una revisión de la normatividad frente a las exigencias, las regulaciones o los acompañamientos por parte de los entes nacionales para con los programas de formación docente.

Con el desarrollo de este trabajo se espera el fortalecimiento de las líneas de investigación de cada una de las licenciaturas, con lo cual se recibe un aporte en el tema de la formación docente como objeto que vincula la investigación con los programas formativos. Por otro lado, el desarrollo de esta investigación posibilitaría el diálogo entre las dos licenciaturas, y nos motivaría al abordaje de debates sobre la formación docente en la facultad y, por qué no, a incluir este tema en las acciones por desarrollar para los procesos de autoevaluación tanto de los programas como de la facultad.

\section{OBJETO}

El grupo de investigación Estudios en Educación y Experiencia Corporal ha desarrollado investigaciones relacionadas con el conocimiento de docentes y estudiantes acerca de las representaciones sociales de sus competencias profesionales, sobre: las Representaciones Sociales del concepto de experiencia corporal ${ }^{6}$. El desarrollo de estas investigaciones ha aportado en la comprensión de aspectos relevantes para la formación específica de los docentes y los estudiantes de Educación Física. Por otro lado, el grupo Lúdica, cuerpo y sociedad ha desarrollado investigaciones sobre la relación entre recreación y escuela ${ }^{7}$, orientadas a la comprensión de posibilidades de acción de la recreación y de un licenciado en este campo, en el espacio escolar.

6 Representaciones sociales de las competencias profesionales del licenciado en educación física de la Universidad Pedagógica Nacional, investigación financiada por el CIUP, vigencia 2011-2012; y Representaciones sociales sobre el concepto de experiencia corporal, que tienen los estudiantes de los semestres de sistematización del Proyecto Curricular Licenciatura Educación Física, financiada por el CIUP vigencia 2013-2014.

7 Pedagogía y metodología de la recreación en la escuela. Investigación financiada por el CIUP, vigencias 2010, 2011 y 2013.
Para los integrantes de los grupos que desarrollan esta investigación, el término formación docente se ha entendido como uno de los temas importantes dentro de cada programa curricular, dadas las características de la misma universidad y los postulados de formación de licenciados que como institución de educación superior debe cumplir. Esta investigación puede, además, contribuir aportando fundamentos disciplinares para los programas de la facultad. Con la participación en la convocatoria interna de la UPN-CIUP, la pretensión de quienes desarrollamos la investigación es realizar hallazgos que permitan seguir argumentando las implicaciones de la formación docente, elemento que resulta común no solo para estos dos programas, sino para las licenciaturas de la facultad.

Con lo anterior, podemos decir que partimos de reconocer lo que se ha desarrollado hasta el momento en cada licenciatura; además, proponemos conocer y estudiar lo que desde los entes de vigilancia y supervisión del país se propone cuando de formación docente se habla. Para dar cuenta de esto, una acción es identificar cuáles son las leyes, los decretos y las resoluciones que han venido reglamentando los programas de formación de docentes.

El objetivo general del presente proyecto es establecer un marco conceptual sobre formación docente adecuado a los desarrollos y las proyecciones del programa Licenciatura en Recreación y Turismo y del Proyecto Curricular Licenciatura en Educación Física generando un proyecto de investigación que facilite la comprensión de este concepto, relacionado con situaciones concretas de los programas. Sobre este propósito se definen los siguientes objetivos específicos:

1. Establecer los antecedentes conceptuales que han construido las líneas de investigación vinculadas al proyecto, así como otros antecedentes documentales sobre formación docente en los programas y la facultad.

2. Indagar las posibilidades de investigación que concreten los desarrollos conceptuales en situaciones específicas de los programas vinculados a la investigación.

3. Construir documentos de conceptualización, con las perspectivas de líneas, grupos, programas y facultad que describan apuestas teóricas sobre formación docente y su proyección. 
4. Formular dos proyectos de investigación que sinteticen las construcciones conceptuales en situaciones específicas de los programas vinculados, según sus campos.

5. Revisar documentos teóricos y legales sobre formación docente que, junto con los antecedentes, permitan la construcción de categorías de estudio y desarrollo conceptual. Para el caso concreto del presente escrito, nos dedicaremos a esta indagación.

\section{MARCO TEÓRICO}

El marco teórico se plantea desde varios ejes. Uno de ellos, y del que hablaremos en este escrito, es la normatividad nacional en relación con la formación docente. Para abordar la normatividad es necesario retomar el significado de norma. Según Comte Sponville,

nos dice lo que debe ser, o permite juzgar lo que no es, se puede valer como sinónimo de la regla, de ideal, pero si se desea darle un sentido más preciso, habrá que insistir en su generalidad: la norma es el género común, cuyas diferentes especies son las reglas, los ideales y los valores. (Comte, 2001, p. 374)

Al adentrarnos en la normatividad, se dice que esta fija normas, o se deriva de ellas o las supone, que enuncia un juicio de valor o depende de él. "Lo normativo se opone a lo descriptivo, se contenta con establecer hechos o explicar lo que proporciona las causas o las razones" (Comte, 2001, pp. 374-375). Por otro lado, se dice que "las normas son comparables con las leyes, pero no todas las leyes son normas" (Ferrater Mora, 2004, p. 2583). Para nuestro interés, se puede decir que las normas que reglamentan la formación docente son normas consideradas legales, pues se proponen reglamentar la formación de los docentes como hechos sociales o institucionales; la normatividad establecida para regular la formación docente debe ser tenida en cuenta y de obligatorio cumplimiento por parte de las instituciones de educación superior que tienen como misión formar a docentes. En este apartado hicimos una revisión de las diferentes leyes, decretos y resoluciones que a lo largo de los últimos años han venido reglamentando la formación docente.

\section{MÉTODO}

Se plantea el análisis de contenido como método para clasificar, ordenar e interpretar la información recolectada en los documentos pretendiendo una comprensión del concepto de formación docente desde referentes y antecedentes específicos de la Facultad. Las estrategias presentadas por el análisis de contenido - a saber, los niveles superficial, analítico e interpretativo- (Quintero \& Ruiz, 2004, p. 107 ) sirven de referente para teorizar la formación docente en el programa Licenciatura en Recreación y Turismo y en el Proyecto Curricular Licenciatura en Educación Física, de la Universidad Pedagógica Nacional.

\section{AVANCES}

Teniendo en cuenta la metodología propuesta para el desarrollo de la investigación, se establecen los siguientes documentos como fuente para dicho desarrollo:

- Documentos maestros de los programas: son los documentos que rigen el desarrollo de las licenciaturas en todos los aspectos. Son los aprobados por el Ministerio de Educación Nacional para la obtención del Registro Calificado.

- Documentos de fundamentación de las líneas de los programas: son documentos de reflexión en los que se postulan lineamientos teóricos y metodológicos para el desarrollo de las líneas de investigación.

- Revistas de la facultad: se refieren a la producción en publicaciones seriadas originadas en la facultad. Específicamente, se refieren a la revista Lúdica Pedagógica, indexada en Colciencias, y a varias revistas de tipo estudiantil que usualmente han recopilado sistematizaciones y trabajos de grado de estudiantes del programa Licenciatura en Educación Física.

- Documentos del Ministerio de Educación Nacional: son documentos publicados por esta entidad, tales como: los decretos, las leyes y las resoluciones pertinentes a la formación docente, las orientaciones pedagógicas para la enseñanza de educación física, recreación y deportes, y otros lineamientos de reflexión o análisis que contengan como eje central la formación docente. 
- Documentos de Coldeportes u otros organismos estatales: cartillas, investigaciones o lineamientos producidos por dichas entidades de carácter estatal, referidos a la formación docente.

- Bases de datos científicas: son documentos que provendrán de la consulta de las bases de datos a las que podamos acceder. Se destacan en estas bases los artículos de investigación científica en el tema de la formación docente que tengan información completa de objetivos, metodología, resultados y conclusiones.

- Otros documentos de instituciones no gubernamentales: son documentos de reflexión o teorización de instituciones cercanas a los campos específicos de la educación y la recreación, tales como la Fundación Colombiana de Tiempo Libre y Recreación (Funlibre), la Asociación Colombiana de Universidades (ASCUN), la Asociación Colombiana de Facultades de Educación (ASCOFADE), la Asociación Red Colombiana de Facultades de Deportes, Educación Física y Recreación (ARCOFADER) y la Federación Colombiana de Educadores (FECODE).

- Otros documentos producidos en los programas o la facultad: son documentos de reflexión o de teorización no publicados, producto del trabajo de docentes o de estudiantes de la facultad.

- Libros y artículos sobre formación de maestros, a escala tanto nacional como internacional.

Cabe tener en cuenta que para este escrito solo se retomará la categoría Fuentes que permitan evidenciar la normatividad que rige la formación docente, ya sea que esta haya sido expedida por la Presidencia de la República, el Congreso de la República o el Ministerio de Educación Nacional.

Una vez ubicada la información, el ejercicio que sigue es su ordenamiento por años, su clasificación y su análisis, lo cual posibilitará la creación de categorías para tener en cuenta en el estudio de la información.

\section{UBICACIÓN DEL CONTEXTO POLÍTICO Y CULTURAL}

Para 1998, la demanda de la educación superior crece y se diversifica, pues la educación superior incluye un sinnúmero de carreras que deben contemplar nuevos conocimientos y nuevas competencias, las cuales deben ser impartidas por instituciones de educación superior. Pero estas, a su vez, se ven enfrentadas a diferentes desafíos y dificultades, como la financiación, la igualdad de condiciones de acceso y permanencia, la capacitación de profesores, la mejora en la calidad de la enseñanza, la investigación, la pertinencia de los planes de estudio y los retos que implican las nuevas tecnologías. La anterior información fue extraída de la Conferencia mundial sobre la educación superior (UNESCO, 1998).

Todo lo anterior hace más notoria la disparidad en nuestro país, en el cual no es fácil el acceso a la educación superior, dadas las diferencias socioeconómicas que aún se viven. Pero la UNESCO propone el intercambio de conocimientos, la cooperación internacional y las nuevas tecnologías que pueden contribuir con nuevas oportunidades de reducir esta disparidad. En el documento Cambio y desarrollo en la educación superior (1998), la entidad propone la misión y las funciones de la educación superior, en pro del desarrollo sostenible y del mejoramiento del conjunto de la sociedad.

Tomando en cuenta lo anterior, el gobierno nacional, en 1998, inicia un proceso dentro de los marcos legislativo, político y financiero, y el cual lo conduce a la reforma de la educación superior, de conformidad con la Declaración Universal de Derechos Humanos, según la cual la educación superior deberá ser accesible a todos. Es a partir de ese momento cuando en Colombia se da inicio a una serie de normas que reglamentan la educación superior y a las instituciones que la imparten.

\section{DOCUMENTOS QUE REGLAMENTAN LA EDUCACIÓN SUPERIOR}

Con base en las distintas fuentes que nos propusimos revisar, a continuación, presentamos los hallazgos obtenidos dentro de la normatividad existente en nuestro país para regular los programas de formación docente.

Podemos hacer una primera mirada desde las diferentes normas que reglamentan los programas de formación docente, para lo cual se tuvo en cuenta hacerla de forma cronológica, partiendo del Decreto 272 de 1998,

por el cual se establecen los requisitos de creación y funcionamiento de los programas académicos de pregrado y postgrado en Educación ofrecidos por las instituciones de educación superior y por las instituciones universitarias, se establece la 
nomenclatura de los títulos y se dictan otras disposiciones. (...) Que de conformidad con los artículos $1^{\circ}$ y 110 de la Ley 115 de 1994, la educación es un proceso de formación permanente, personal, cultural y social que se fundamenta en una concepción integral de la persona humana, de su dignidad, de sus derechos y de sus deberes, que requiere un educador de reconocida idoneidad moral, ética, pedagógica y profesional. Que de acuerdo con el artículo 4을 de la Ley 115 de 1994, el Estado deberá atender en forma permanente los factores que favorecen la calidad y el mejoramiento de la educación y especialmente velar por la cualificación y formación de los educadores. Que los programas en el área de Educación, según lo establecido en el artículo 113 de la Ley 115 de 1994, deben estar acreditados en forma previa. Que de acuerdo con el artículo 36 de la Ley 30 de 1992 es función del Consejo Nacional de Educación Superior, CESU, proponer al Gobierno Nacional la reglamentación y procedimientos sobre los requisitos de creación y funcionamiento de los programas académicos, establecer la nomenclatura de los títulos y definir los mecanismos para evaluar la calidad académica de la educación superior y de sus programas.

En abril de 2002, entra en vigencia el Decreto 808, el cual considera:

Que la Educación Superior es un servicio público esencial de naturaleza cultural con una función social que le es inherente y, que como tal corresponde al Estado ejercer el fomento y la inspección $\mathrm{y}$ vigilancia, en procura de garantizar la calidad y la eficiencia en su prestación, y de facilitar el acceso de las personas aptas a la Educación Superior. Que en el ejercicio de la Inspección y Vigilancia de la Educación Superior corresponde al Presidente de la República fomentar la calidad de los programas académicos de las Instituciones de Educación Superior, a través de procesos de evaluación de la calidad, dentro de los cuales la medida de tiempo de trabajo académico estudiantil es un mecanismo e indicador esencial. Que de conformidad con la Ley 30 de 1992, el fomento de la Educación Superior debe estar orientado, entre otros, a facilitar la interacción y circulación de los actores y activos académicos de las Instituciones de Educación Superior, estimulando la cooperación entre ellas y de estas con la comunidad internacional. Que es necesario reglamentar mecanismos que faciliten la movilidad de estudiantes, la homologación de estudios, y la convalidación de títulos de programas académicos cursados en el exterior, adoptando una medida compatible con la más utilizada internacionalmente. Que dentro de los mecanismos de transferencia estudiantil, es necesaria la adopción de una medida de tiempo de trabajo académico que permita homologar y reconocer los logros alcanzados por los estudiantes en sus actividades académicas, que pueda ser utilizada de manera flexible por las diferentes Instituciones de Educación Superior del país, de tal forma que sirva de parámetro para hacer efectiva la transferencia de estudiantes.

El Decreto 808 de 2002 fue derogado mediante la promulgación del Decreto 2566 de 2003, "Por el cual se establecen las condiciones mínimas de calidad y demás requisitos para el ofrecimiento y desarrollo de programas académicos de educación superior y se dictan otras disposiciones". Este decreto considera:

Que la educación superior es un servicio público de carácter cultural con una función social que le es inherente y, que como tal, de acuerdo con el Artículo 67 de la Constitución Política y el Artículo 3 de la Ley 30 de 1992, le corresponde al Estado velar por su calidad, por el cumplimiento de sus fines mediante el ejercicio de la inspección y vigilancia y mantener la regulación y el control sobre ella. Que de acuerdo al Artículo 31 de la Ley 30 de 1992, le corresponde al Presidente de la República propender por la creación de mecanismos de evaluación de la calidad de los programas académicos de educación superior. Que de conformidad con el Artículo 32 de la Ley 30 de 1992, la suprema inspección y vigilancia de la educación, se ejerce a través de un proceso de evaluación, para velar por su calidad, el cumplimiento de sus fines, la mejor formación moral, intelectual y física de los educandos y por la adecuada prestación del servicio. Que los programas en el área de educación, según lo establecido en el Artículo 113 de la Ley 115 de 1994, deben estar acreditados en forma previa. Que el Artículo 8 de la Ley 749 de 2002 dispone que para poder ofrecer y desarrollar un programa de formación técnica profesional, tecnológica, y profesional de pregrado o de especialización, nuevo o en funcionamiento, se requiere obtener registro calificado del misino, correspondiendo al Gobierno Nacional su reglamentación. Que corresponde al Presidente de la República expedir los decretos necesarios para la cumplida ejecución de las leyes.

Posteriormente, fue expedida la Resolución 1036 de 2004, la cual define "las características específicas de calidad para los programas de pregrado y especialización en Educación":

Que mediante Decreto 2566 del 10 de septiembre de 2003 el Gobierno Nacional reglamentó las condiciones mínimas de calidad y demás requisitos para el ofrecimiento y desarrollo de programas académicos de educación superior. Que conforme a lo dispuesto en los artículos 1 y 4 del 
Decreto 2566 del 10 de septiembre de 2003, le corresponde al Ministerio de Educación Nacional fijar las características específicas de calidad para cada programa con el apoyo de las instituciones de educación superior, las asociaciones de facultades o profesionales o de pares académicos. Que en la definición de las características específicas de calidad para el ofrecimiento y desarrollo de programas de formación profesional de pregrado y especialización aplicables para los programas en el área de Educación, se contó con la participación activa de la comunidad académica nacional de la respectiva área de conocimiento, en los encuentros organizados en los seis Centros Regionales de Educación Superior (CRES) y en otros escenarios académicos; logrando en su definición altos niveles de consenso y un mayor compromiso con la calidad de la Educación Superior. Que por lo anterior se hace necesario definir las características específicas de calidad aplicables a los programas de pregrado y especialización en Educación.

En 2008 aparece la Ley 1188, "por la cual se regula el registro calificado de programas de educación superior y se dictan otras disposiciones":

Para poder ofrecery desarrollar un programa académico de educación superior que no esté acreditado en calidad, se requiere haber obtenido registro calificado del mismo. El registro calificado es el instrumento del Sistema de Aseguramiento de la Calidad de la Educación Superior mediante el cual el Estado verifica el cumplimiento de las condiciones de calidad por parte de las instituciones de educación superior. Compete al Ministerio de Educación Nacional otorgar el registro calificado mediante acto administrativo debidamente motivado, en el que se ordenará la respectiva incorporación en el Sistema Nacional de Información de la Educación Superior, SNIES, y la asignación del código correspondiente. Condiciones de calidad: Para obtener el registro calificado de los programas académicos, las instituciones de educación superior deberán demostrar el cumplimiento de condiciones de calidad de los programas y condiciones de calidad de carácter institucional. Esta Ley deroga el artículo 1 del Decreto 2566 de 2003.

En 2010 se promulga el Decreto 1295, el cual derogó el Decreto 2566 de 2003, y mediante el cual "se reglamenta el registro calificado de que trata la ley 1188 de 2008 y la oferta y desarrollo de programas académicos de educación superior". En dicho decreto se establece que "las instituciones de educación superior definirán la organización de las actividades académicas de manera autónoma" y estable, y se mantendrán los créditos académicos como "unidad de medida del trabajo académico". También define las condiciones para obtener registro calificado, las condiciones de calidad y "las actividades de investigación que permitan desarrollar actitudes críticas y capacidades creativas para proponer alternativas para el avance de la ciencia, la tecnología, las artes o las humanidades; además, propone una cultura de autoevaluación" (Guacaneme, 2011, p. 66).

En 2010 se promulga la Resolución 5443, la cual deroga la Resolución 1036 de 2004 y “define las características específicas de calidad de los programas de formación profesional en educación, en el marco de las condiciones de calidad, y se dictan otras disposiciones". Dicha resolución considera:

Que formar a los educadores, como agentes del sistema educativo que prioritariamente contribuyen a mejorar la calidad de vida en el país, como profesionales que apoyan y acompañan a los niños, niñas, jóvenes y adultos en sus procesos de desarrollo personal y de aprendizaje, corresponde a los programas de formación.

Exige, además, quelosformadores deprofesores posean las competencias básicas y profesionales correspondientes, y las cuales deben ser promovidas en sus estudiantes. Por otra parte, ordena incluir el uso de las tecnologías de la información y la comunicación (TIC) en el desarrollo de los programas de formación. Se ratifica, así mismo, la necesidad de tener un plan de autoevaluación para plantear un programa que cualifique los aspectos de calidad evidenciándolos como oportunidades de mejoramiento. También da a los normalistas graduados la posibilidad de acceder a los programas de licenciatura.

En agosto de ese mismo año se expide la Resolución 6966, que modifica los artículos 3 y 6 de la Resolución 5443 , los cuales hablan de la denominación de los programas y las prácticas pedagógicas. En cuanto a la denominación, la Resolución 6966 establece que la Institución de Educación Superior, IES, debe definir la denominación del programa en coherencia con su propósito. En lo que concierne a la formación de docentes, retoma lo establecido en la Ley 115 de 1994: para el nivel de preescolar se debe tener en cuenta la Ley 1098 de 2006 (Código de Infancia y Adolescencia), y para la formación de docentes del ciclo de primaria, la Ley 1297 de 2009, la cual establece que para ejercer como maestros en primaria, el título de normalista o de licenciado 
en educación no requiere ningún énfasis en el área de conocimiento; respecto a los profesores de educación básica y media, no hace modificaciones.

En 2012 se promulga la Resolución 5290, "por la cual se reorganiza la Comisión Nacional Intersectorial de Aseguramiento de la Calidad de la Educación Superior (CONACES)", conformada por el Ministerio de Educación Nacional y por el director del Departamento de Ciencias de la Tecnología e Innovación (Colciencias):

Lo anterior sin prejuicio de que puedan ser convocados los representantes de los organismos asesores del Gobierno Nacional en materia de educación superior y de la academia.

Que la Ley 1188 de 2008 asigna funciones de evaluación de la calidad en educación superior a la Comisión Nacional Intersectorial para el Aseguramiento de la Calidad de la educación Superior (Conaces).

Que con el fin de mejorar y agilizar los procesos de Aseguramiento de la calidad de la educación superior se hace necesario modificar la organización de la Comisión Nacional Intersectorial de Aseguramiento de la Calidad de la Educación Superior (Conaces).

Según el Decreto 2219 de 2014, el cual reglamenta el ejercicio de inspección y vigilancia de la Educación Superior, el Ministerio puede, en desarrollo de una labor de seguimiento académico y administrativo de la IES que incumpla las normas, suspender registros y ordenar la creación de una fiducia para manejo de bienes, entre otros aspectos:

Que por disposición del artículo 67 de la Constitución Política, la educación es un derecho de la persona y un servicio público que tiene una función social; con ella se busca el acceso al conocimiento, a la ciencia, a la técnica, y a los demás bienes y valores de la cultura. Que el mismo artículo 67 de la Constitución señala que le corresponde al Estado regular y ejercer la suprema inspección y vigilancia de la educación con el fin de velar por su calidad, por el cumplimiento de sus fines y por la mejor formación moral, intelectual y física de los educandos; garantizar el adecuado cubrimiento del servicio y asegurar a los menores las condiciones necesarias para su acceso y permanencia en el sistema educativo. Que el artículo 69 de la Constitución garantiza la autonomía universitaria, en virtud de la cual las universidades podrán darse sus directivas y regirse por sus propios estatutos, de acuerdo con la ley. Que de conformidad con los artículos 67 y 189 -numerales 21, 22 y 26 de la Constitución Política, y el artículo 31 de la Ley 30 de 1992, le corresponde al Presidente de la República ejercer la suprema Inspección y Vigilancia de la educación, con el fin de velar por su calidad, por el cumplimiento de sus fines, y por la mejor formación moral, intelectual y física de los educandos, y la correcta inversión de los recursos; función que fue delegada por el Presidente de la República al Ministro de Educación Nacional mediante el Decreto 698 de 1993, de conformidad con el artículo 33 de la Ley 30 de 1992. Que la Ley 30 de 1992, "Por la cual se organiza el servicio público de la educación superior", consagra en su artículo $2^{\circ}$ que la educación superior es un servicio público cultural, inherente a la finalidad social del Estado, y en su artículo $3^{\circ}$ garantiza la autonomía universitaria, correspondiéndole al Estado velar por la calidad del servicio educativo a través del ejercicio de la suprema inspección y vigilancia de la educación superior. Que el artículo $6^{\circ}$ de la Ley 30 de 1992 dispone que uno de los objetivos de la educación superior y de sus instituciones es prestar a la comunidad un servicio con calidad, el cual hace referencia a los resultados académicos, a los medios y procesos empleados, a la infraestructura institucional, a las dimensiones cualitativas y cuantitativas del mismo y a las condiciones en que se desarrolla cada institución. Que la Ley 30 de 1992 señala en su artículo 28 alcance la autonomía universitaria y reconoce a las Instituciones de Educación Superior derecho a arbitrar y aplicar sus recursos para el cumplimiento su misión social y su función institucional. Que en virtud del literal e) del artículo de la Ley 30 de 1992, la suprema inspección y vigilancia de la educación superior implica velar porque en las instituciones privadas educación superior, constituidas como personas jurídicas de utilidad común, sus rentas se conserven y se apliquen debidamente y que en todo lo esencial se cumpla con la voluntad de sus fundadores.

La Ley 1740 de 2014 es la ley,

Por la cual se desarrolla parcialmente el artículo 67 y los numerales 21,22 y 26 del artículo 189 de la constitución política, se regula la inspección y vigilancia de la educación superior, se modifica parcialmente la Ley 30 de 1992 y se dictan otras disposiciones.

El artículo 1 del capítulo I de dicha ley se refiere a su finalidad, la cual establece las normas de inspección y vigilancia de la Educación superior en Colombia, con el fin de velar por la calidad de ese servicio público, su continuidad, la mejor formación moral, intelectual y física 
de los educandos, entre otras; para garantizar todo ello, la ley adiciona los siguientes literales al artículo 31 de la ley 30 de 1992: a) Velar por la calidad y la continuidad del servicio público de educación superior; b) Propender por la mejor formación moral, intelectual y física de los educandos y por el cumplimiento de los objetivos de la educación superior; c) Velar por el adecuado cubrimiento del servicio público de educación superior; d) Que en las instituciones privadas de educación superior, constituidas como personas jurídicas de utilidad común, sus rentas se conserven y se apliquen debidamente, y que en todo lo esencial se cumpla con la voluntad de sus fundadores, sin que pueda consagrarse o darse de forma alguna el ánimo de lucro; e) Que en las instituciones oficiales de educación superior se atienda a la naturaleza de servicio público cultural y a la función social que les es inherente, se cumplan las disposiciones legales y estatutarias que las rigen y que sus rentas se conserven y se apliquen debidamente.

Esta ley, además, propone otros 19 artículos, los cuales tienen por objeto velar por el funcionamiento de las instituciones de educación superior. A continuación se hace el listado de los 24 artículos restantes: el artículo $2^{\circ}$ hace referencia a la prevención; el artículo $3^{\circ}$, a los objetivos de la inspección y la vigilancia; el artículo $4^{\circ}$, al ámbito de aplicación; el artículo $5^{\circ}$, a facultades generales; el artículo $6^{\circ}$, a inspección; el artículo $7^{\circ}$, a funciones de inspección; el artículo $8^{\circ}$, a vigilancia; el artículo $9^{\circ}$, a funciones de vigilancia; el artículo $10^{\circ}$, a medidas preventivas; el artículo 11, a vigilancia especial; el artículo 12, al procedimiento para la adopción de medidas preventivas y de vigilancia especial; el artículo 13, a medidas de vigilancia especial; el artículo 14, a los institutos de salvamento para la protección temporal de recursos y bienes en el marco de la vigilancia especial; el artículo 15, a la acción revocatoria y de simulación para la protección de los bienes de la institución de educación superior; el artículo 16, a la cesación de actividades no autorizadas; el artículo 17 a sanciones; el artículo 18, a la aplicación de sanciones; el artículo 19, a los criterios para graduar la sanción; el artículo 20, a investigación preliminar; el artículo 21, a la continuidad del derecho a la educación; el artículo 22, a los trámites ante el Ministerio de Educación Nacional; el artículo 23, a los trámites para la Superintendencia de Educación; el artículo 24: transitorio, artículo 25: derogatorio.

\section{CATEGORIIAS DESARROLLADAS}

Una vez organizada y analizada la información referida anteriormente, las categorías que surgen son: formación y calidad, por ser estas incluidas en las normas que se trabajaron en el presente artículo.

La formación es relevante en cada una de estas normas. A continuación, se presentan algunos apartes en los que aparece dicho término:

- La importancia de la formación moral, intelectual y física de los educandos y de la adecuada prestación del servicio.

- Para poder ofrecer y desarrollar un programa de formación técnica profesional, tecnológica, y profesional de pregrado o de especialización, nuevo o en funcionamiento, se requiere obtener registro calificado.

- Se definen las características específicas de calidad de los programas de formación profesional en educación dentro del marco de las condiciones de calidad y se dictan otras disposiciones.

- Exige que los formadores de profesores posean las competencias básicas y profesionales, las cuales deben ser promovidas en sus estudiantes, y que se incluya el uso de las TIC en el desarrollo de los programas de formación.

- Se debe definir la denominación del programa en coherencia con el propósito de este; para lo concerniente a la formación de docentes, retoma lo establecido en la Ley 115 de 1994: para el nivel de preescolar se debe tener en cuenta la Ley 1098 de 2006 (Código de Infancia y Adolescencia), y para la formación de docentes del ciclo de primaria, la Ley 1297 de 2009.

- El artículo 67 de la Constitución señala que le corresponde al Estado regular y ejercer la suprema inspección y vigilancia de la educación, con el fin de velar por su calidad, por el cumplimiento de sus fines y por la mejor formación moral, intelectual y física de los educandos; también, garantizar el adecuado cubrimiento del servicio y asegurar a los menores las condiciones necesarias para su acceso y permanencia en el sistema educativo. 
El otro término relevante es la calidad. Los decretos, las resoluciones y las leyes que reglamentan la educación superior tienen en común la búsqueda de la calidad, que, a su vez, se refiere a:

- Atender de forma permanente los factores que favorecen la calidad y el mejoramiento de la educación, así como definir los mecanismos para evaluar la calidad académica. Ello, teniendo en cuenta que la educación superior es un servicio público esencial de naturaleza cultural con una función social que le es inherente, y que, como tal, le corresponde al Estado ejercer su fomento, su inspección y su vigilancia, en procura de garantizar la calidad y la eficiencia en su prestación.

- Es deber del Estado fomentar la calidad de los programas académicos de las instituciones de educación superior.

- Establecer las condiciones mínimas de calidad y los demás requisitos para el ofrecimiento y el desarrollo de programas académicos de educación superior, el cual, a su vez, se verifica mediante el cumplimiento del registro calificado.

- Formar a los educadores como agentes del sistema educativo que prioritariamente contribuyen a mejorar la calidad de vida en el país, como profesionales que apoyan y acompañan a los niños, las niñas, los jóvenes y los adultos en sus procesos de desarrollo personal y de aprendizaje, corresponde a los programas de formación.

- Se ratifica la necesidad de tener un plan de autoevaluación para plantear un programa que cualifique los aspectos de calidad evidenciándolos como oportunidades de mejoramiento. Se da la posibilidad a los normalistas graduados para accedan a los programas de licenciatura.

- Se reorganiza la Comisión Nacional Intersectorial de Aseguramiento de la Calidad de la Educación Superior (CONASES).

\section{CONCLUSIONES}

Durante los últimos 16 años, la Presidencia de la República, el Congreso de la República y el Ministerio de Educación Nacional han sancionado cinco decretos, cuatro resoluciones y dos leyes con el propósito de normar, supervisar y garantizar la formación de los docentes. Estas normas pueden leerse desde dos enfoques: el primero de ellos hace referencia al funcionamiento, y da la apariencia de ser esto lo central de la norma; el segundo se refiere a la calidad expresada en la autonomía. Con ello, no deja de ser problemático el asunto que se origina con el término "calidad", pues parece abrirse una brecha entre lo autorizado y lo de calidad, que deja ver niveles entre las instituciones y, por otro lado, expone una incoherencia esencial: ¿lo autorizado no es de calidad, aunque es lo regulado estatalmente?

También aparecen problemáticas sentidas, como lo es el asunto de la autonomía de las universidades públicas en medio del desfinanciamiento; más específicamente, respecto a las universidades formadoras de docentes, $\mathrm{ni}$ esta autonomía y ni esta calidad parecen ser tan ciertas, dadas las regulaciones homogéneas de la profesión docente. No se contempla en estas normas el hecho que se propuso luego de la reforma de la Constitución de 1991 y desde la Ley 115, en lo relativo a establecer la pedagogía como el saber fundante de la formación de maestros para ser asumida como una formación profesional (Calvo, 2004).

Se identifica en los documentos revisados que la normatividad analizada no hace referencias específicas a las instituciones de educación superior encargadas de la formación de docentes; es decir, no se evidencian en ellas exigencias directas sobre lo que deben contener los programas de formación desde una visión o un perfil que se ocupe de la formación en pro de lo que se vive en la actualidad. Las normas estudiadas pretenden homogenizar desde lo administrativo, lo cual permite pensar que el tema de la misión de educar se deja al libre albedrio de lo que cada institución de educación superior quiera o entienda como importante en el proceso formativo.

Si bien a los entes de control les interesa la educación de calidad, esta se encuentra referida a unas condiciones particulares desde las posibilidades que cada institución de educación superior debe resolver desde los procesos de autoevaluación que hace cada programa; es el Ministerio de Educación Nacional el que establece si un programa puede o no seguir con la oferta de dichos procesos; es decir, son las propias instituciones las encargadas de autoevaluarse objetivamente para dar cuenta de su propio funcionamiento. 
En referencia a las categorías desarrolladas, cabe anotar que el término calidad resulta ambiguo: en las normas se incluye, pero en ninguna de ellas se expresa claramente a qué se refiere este término en la educación superior; se da por entendido, podría pensarse, que todos los relacionados con la educación saben bien lo que este término significa, cosa que no es tan cierta, pues el término calidad es subjetivo y tiene relación con lo que cada institución de educación superior cuenta. Y con el término formación pasa algo similar, dado que en las normas no hay referencias explícitas a él: por ejemplo, no se ubicó ningún documento que tenga en cuenta las diferentes áreas del conocimiento y, como consecuencia de ello, proponga o establezca de manera específica referentes de desarrollo para ser tenidos en cuenta dentro de los procesos de formación de docentes.

Para el desarrollo de esta investigación, ubicamos la normatividad revisada en la precategoría de políticas, teniendo en cuenta que son estas normas las que han posibilitado a las instituciones de educación superior crear y mantener programas de formación docente bajo la mira de los entes estatales encargados de supervisar y controlar unos mínimos para todas las instituciones que tienen por objeto formar a docentes.

En cuanto a los vínculos que se pueden hacer desde las dos licenciaturas, esta investigación delinea algunos campos posibles de desarrollar, que les apuestan, en conjunto, a la calidad y a la formación, tales como el desarrollo del potencial humano y el bienestar, como elementos fundantes de la vida en sociedad.

\section{REFERENCIAS}

Calvo, G. (2004). La formación de los docentes en Colombia. Bogotá: Ascun-Unesco.

Comte, A. (2001). Diccionario Filosófico, París: Paidós, 374-375.

Decreto No. 272 de 1998. Presidencia de la República, Colombia.

Decreto No. 808 de 2002. Presidencia de la República, Colombia.

Decreto No. 2566 de 2003. Presidencia de la República, Colombia.

Decreto No. 1295 de 2010. Presidencia de la República, Colombia.

Decreto No. 2219 de 2014. Presidencia de la República, Colombia.

Guacaneme, E., Bautista, M. \& Salazar, C. (2011). El contexto normativo de formulación de los programas de formación inicial de profesores de matemáticas. Voces y silencios. Revista latinoamericana de Educación, 2, (1), 62-77.

Ferrater Mora,J. (2004). Diccionario de filosofía. Barcelona: Ariel Filosofía.

Ley No. 1188 de 2008. Congreso de la República, Colombia.

Ley No. 1740 de 2014. Congreso de la República, Colombia.

Quintero, M. \& Ruiz, A. (2004). ¿Qué significa investigar en educación? Bogotá: Universidad Distrital Francisco José de Caldas.

Resolución No. 1036 de 2004. Ministerio de Educación Nacional, Colombia.

Resolución No. 6966 de 2010. Ministerio de Educación Nacional, Colombia.

Resolución No. 5290 de 2012. Ministerio de Educación Nacional, Colombia.

Resolución No. 5443 de 2012. Ministerio de Educación Nacional, Colombia.

UNESCO (1998). Declaración mundial sobre la educación superior en el siglo XXI: visión y acción y marco de acción prioritaria para el cambio y el desarrollo de la educación superior. Recuperado de: http://www. unesco.org/education/educprog/wche/declaration spa.htm 